\title{
Ultrathin epitaxial Fe films on vicinal GaAs(001): A study by spin-resolved photoelectron spectroscopy
}

\author{
T. Zhang, ${ }^{\text {a) }}$ M. Spangenberg, ${ }^{\text {a) }}$ and D. Greig \\ Department of Physics and Astronomy, University of Leeds, Leeds LS2 9JT, United Kingdom \\ N. Takahashi \\ Department of Physics, Faculty of Education, Kagawa University, Kagawa 760-8522, Japan
}

T.-H. Shen ${ }^{\text {b) }}$

Joule Physics Laboratory, Institute for Materials Research, University of Salford, Greater Manchester M5 4WT, United Kingdom

J. A. D. Matthew

Department of Physics, University of York, Heslington, York YO1 5DD, United Kingdom

S. Cornelius, M. Rendall, and E. A. Seddon

CLRC Daresbury Laboratory, Warrington, WA4 4AD, United Kingdom

(Received 10 October 2000; accepted for publication 11 December 2000)

\begin{abstract}
Thin epitaxial Fe films have been grown on vicinal $\mathrm{GaAs}(001)$ substrates and their remanent magnetic properties and the degree of substrate atom diffusion investigated using synchrotron-based photoelectron spectroscopy. The vicinal Fe films, though exhibiting greater As diffusion than their singular homologues, displayed better film quality both from the structural and the magnetic points of view. The spin-resolved valence spectra of the vicinal films resemble those for crystalline bulk Fe at lower film thicknesses than for singular films. (C) 2001 American Institute of Physics.
\end{abstract}

[DOI: $10.1063 / 1.1345820]$

The study of metallic films on semiconductors has been the subject of intense interest for a considerable period due to their wide-ranging applications in the electronics industries. ${ }^{1}$ Recently, the growth and characterization of thin ferromagnetic films on semiconductors has been of particular interest, as such structures have potential for "spin-electronic" devices; i.e., devices that combine control of both the charge and the spin of the electron. ${ }^{2}$ As a model system, $\mathrm{Fe}-$ $\operatorname{GaAs}(001)$ has attracted attention, particularly because of its growth behavior. ${ }^{3-6}$ However, while there has been a report on the magnetic anisotropy of Fe films on vicinal $\mathrm{Au}$ and $\mathrm{Ag}$ surfaces, ${ }^{7}$ work on Fe films grown on GaAs substrates has concentrated on singular GaAs surfaces. In this letter, we report an investigation of epitaxial Fe films grown directly on vicinal GaAs(001) substrates, which shows that the presence of steps on the surface significantly influences the properties of the Fe overlayers.

As distinct from singular surfaces, vicinal $\mathrm{GaAs}(001)$ substrates are formed by cutting the crystal a few degrees from an exact low-index orientation, usually towards a specific crystallographic direction. The minimum energy state of such a surface consists of a series of flat terraces, having a precise low index, separated by steps. In the case of a small value of off-cut angle on $\mathrm{GaAs}(001)$, the average step separation is sizable [e.g., about $50 \AA$ for a $3^{\circ}$ "off-cut" GaAs (001)]. While maintaining sizable flat terraces, the presence of the steps is known to promote single-surface

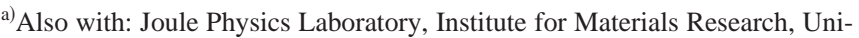
versity of Salford, Greater Manchester, M5 4WT, U.K.

b) Author to whom correspondence should be addressed; electronic mail: t.shen@salford.ac.uk
}

reconstructions. ${ }^{8}$ Homoepitaxial growth on semiconductors maintaining a stepped surface is possible under correct growth conditions. ${ }^{9}$ The quasiperiodicity of the steps has also been exploited to produce quantum wires. ${ }^{10}$ As a first step in the study of $\mathrm{Fe}$ grown on vicinal GaAs surfaces, $\mathrm{GaAs}(001)$ substrates $\left(n^{+}\right.$doped) with $3^{\circ}$ off cut towards (111)A, as well as their singular counterparts, were used in the present study. The vicinal substrates have steps that are perpendicular to the [110] direction, with edges running parallel to the [110] direction. A further $1.5 \mu \mathrm{m}$ epitaxial GaAs layer $(n$ type, with doping concentration $2 \times 10^{18} \mathrm{~cm}^{-3}$ ) was grown by molecular-beam epitaxy (MBE) on both the singular and vicinal substrates. The surface of the substrate was then capped with amorphous As.

Experiments were carried out in a ultra-high-vacuum (UHV) chamber (base pressure better than $3 \times 10^{-10} \mathrm{mbar}$ ) on the undulator line 5U.1 at the SRS Daresbury Laboratory, as well as in the Leeds University MBE chamber. The stepped surface of the substrate was confirmed by reflection high-energy electron diffraction. The amorphous-As layer was removed in UHV, prior to any measurements, by heating the samples. Clean GaAs surfaces with a variety of As $3 d$ to Ga $3 d$ core-level peak intensity ratios were investigated. For simplicity, we label those reported in this work as the following: "sample A" and "sample B" vicinal surfaces with ratios 1.09 and 0.92 at zero Fe coverage; "sample C" and "sample D" singular surfaces with As $3 d / \mathrm{Ga} 3 d$ ratios of 1.12 and 0.97 , respectively. For convenience, the substrates with the higher ratio values (samples A and C) will simply be referred to as "As rich" and those with the lower ratios (samples B and D) as "Ga rich." For singular (001) surfaces, we have reported elsewhere that the evolution of sec- 


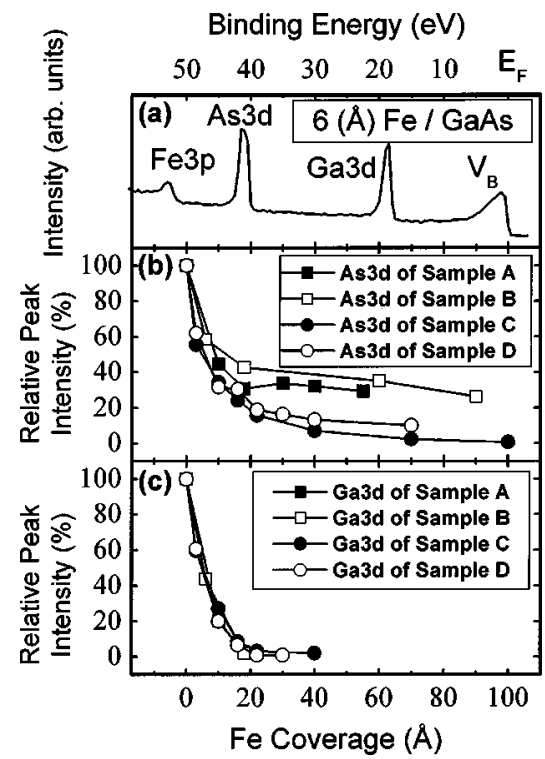

FIG. 1. Spin-integrated core-level photoelectron data. (a) A typical survey spectrum of $6 \AA \mathrm{Fe}$ on a Ga-rich vicinal $\mathrm{GaAs}(001)$ substrate; (b) relative As $3 d$ photoemission peak intensities with Fe coverage; (c) relative Ga $3 d$ photoemission peak intensities with Fe coverage. Samples A, B, C, and D identify the substrates as As-rich vicinal, Ga-rich vicinal, As-rich singular, and Ga-rich singular, respectively.

ondary electron-spin polarization with Fe film thickness is highly dependent on such differences in the substrate surface stoichiometry, notwithstanding the experimental difficulties associated with controlling the value of the ratio precisely. ${ }^{11}$ The sample preparation and Fe deposition conditions are as in that study. In situ low-energy electron diffraction (LEED) was employed to monitor structural ordering at different stages of Fe film deposition.

The incident photon energy was $120 \mathrm{eV}$ and a $-20 \mathrm{~V}$ bias was applied to the sample in order to suppress any stray electrons. A magnetizing current pulse, producing an inplane magnetic field along the [100] direction of the substrates, allowed the polarization measurements to be performed in remanence. The photoelectrons were energy analyzed in a 50-mm-radius hemispherical energy analyzer and spin resolution was achieved with a Mirco-Mott polarimeter ( $20 \mathrm{kV}$ working voltage). The polarimeter is equipped with four independent detectors allowing simultaneous determination of the two transverse polarization components. These directly relate to magnetization in the [100] and [010] directions of the substrate. The effective Sherman function of the Micro-Mott polarimeter, operated at $20 \mathrm{kV}$ and with an energy window of $400 \mathrm{~V}$, was estimated to be 0.12 . Secondary electron-polarization measurements were normally carried out on $0.1 \mathrm{eV}$ kinetic-energy secondary electrons, as the electron count rate at this kinetic energy is very high. For the spin-resolved measurements, the energy resolution was, typically, set to $0.4 \mathrm{eV}$. Both valence-band photoemission and secondary electron emission are much more surface sensitive than magneto-optic Kerr rotations, and the spin polarization of the secondary emission is thus a measure of the surface magnetization.

Figure 1(a) shows a typical spin-integrated survey spectrum of photoelectrons taken from vicinal GaAs with $6 \AA$ nominal thickness of Fe (sample B). The Fe $3 p$, As $3 d$, and $\mathrm{Ga} 3 d$ core levels can be clearly identified. It is also interestGa $3 d$ core levels can be clearly identified. It is also interest- substrate.
Downloaded 21 Sep 2006 to 146.87 .125 .97 . Redistribution subject to AIP licens

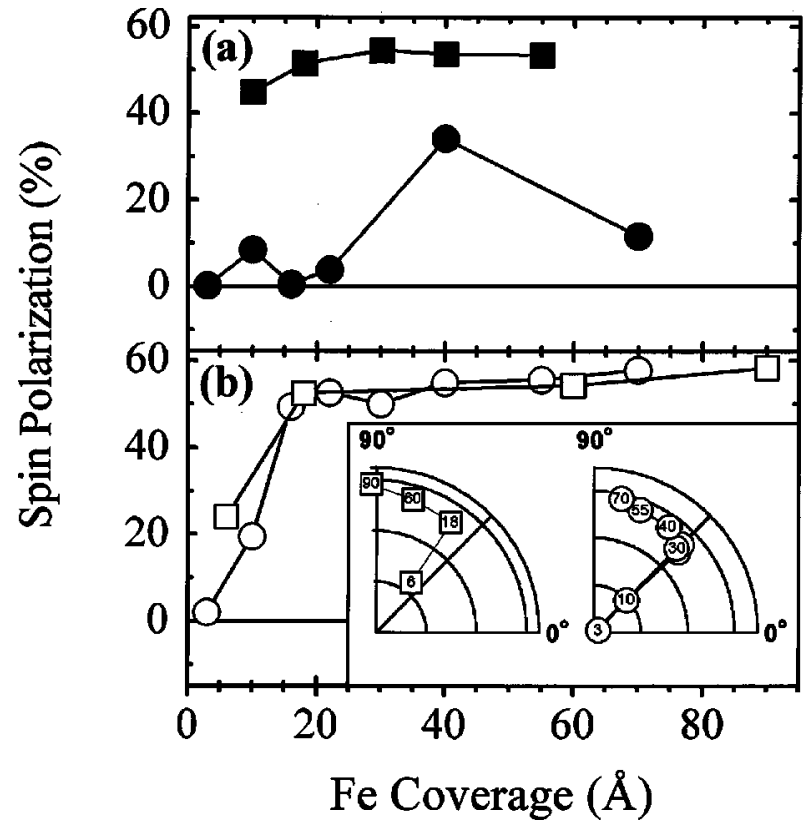

FIG. 2. Spin polarization of secondary electrons as a function of Fe coverage. (a) As-rich substrates and (b) Ga-rich substrates. The squares indicate vicinal substrates (samples A and B) and the circles singular substrates (samples $\mathrm{C}$ and $\mathrm{D}$ ). The inset in (b) shows the rotation of the spinpolarization vector for Ga-rich vicinal and Ga-rich singular substrates. $0^{\circ}$ and $90^{\circ}$ axis directions are along one of the $\langle 100\rangle$ directions of the GaAs. The magnetizing field is along the $90^{\circ}$ axis. Numbers in the symbols indicate Fe coverage in $\AA$.

ing to note that the metallic-like Fermi edge is well defined and the LEED patterns clearly indicate epitaxial growth. Unexpectedly, we also found that the LEED spots were considerably brighter and sharper for the vicinal films than for the singular films (at all Fe thicknesses), clearly indicating that the vicinal films had better structural quality than their singular counterparts. Figures 1(b) and 1(c) illustrate the change in relative peak intensity of the As $3 d$ and Ga $3 d$ core levels with increasing Fe film thickness. In Fig. 1(c), it is obvious that the relative $\mathrm{Ga}$ core-level intensities vary in a very similar manner for all of the samples. In contrast, the As core levels in Fig. 1(b) decay at a much slower rate, indicating substantial As diffusion into the Fe overlayers, and it is clear that the As diffusion is greater in the vicinal films than in the singular films.

Figure 2 summarizes the secondary electron-spinpolarization data for samples A to D. The results for films on As-rich substrates are plotted in Fig. 2(a), and those for films on Ga-rich substrates are plotted in Fig. 2(b). In Fig. 2, the squares are for vicinal substrates and the circles for singular substrates. Films grown on the As-rich and the Ga-rich singular substrates (samples C and D, circles in Fig. 2) exhibit very different polarization behavior. For the As-rich singular case the anomalous behavior is associated with complex domain structure as indicated by measurements of Kerr rotations. ${ }^{11}$ In contrast, the polarization behavior of the two vicinal films (samples A and B, squares in Fig. 2) is very similar. This preliminary finding suggests that either the geometrical influence of the steps and/or the presence of significant amounts of surface As over-rides the destabilizing influence of the initial surface stoichiometry of the GaAs

o AlP license or copyright, see http://apl.aip.org/apl/copyright.jsp 


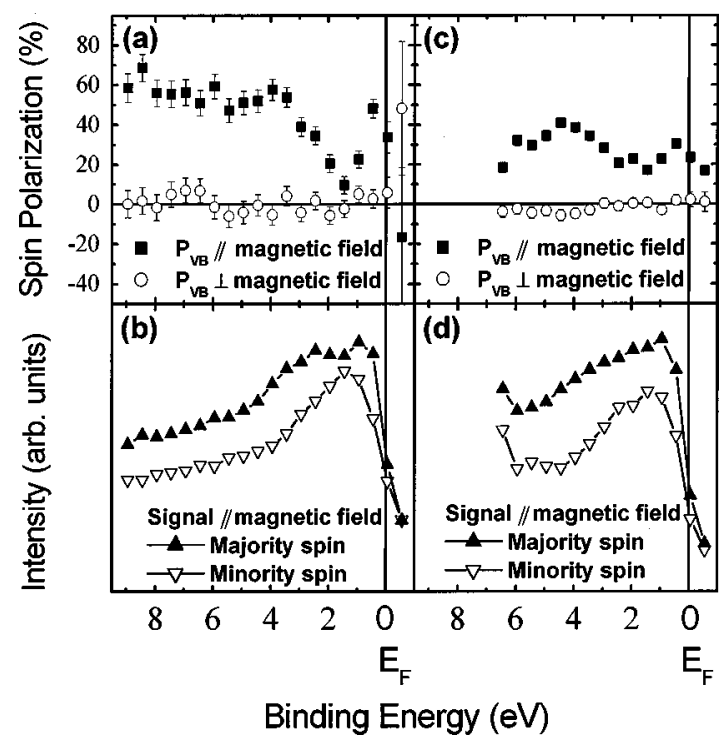

FIG. 3. (a) Spin polarization of valence electrons and (b) spin-resolved valence photoelectron spectra for $90 \AA \mathrm{Fe}$ on Ga-rich vicinal GaAs(001). (c) and (d) are the corresponding data for $80 \AA \mathrm{Fe}$ on Ga-rich singular $\operatorname{GaAs}(001)$

The spin polarization of the secondary electrons at large coverage $(\sim 50 \%-55 \%)$ is much higher than that of the conduction band of pure iron ${ }^{12}$ due to the spin dependence of the mean-free paths of the secondary electrons. ${ }^{13}$ The inset in Fig. 2(b) shows the change in remanent magnetization direction (as revealed by the spin polarization of the secondary electrons) with Fe film thickness on a vicinal and a singular Ga-rich substrate (samples B and D). Both samples exhibit an initial remanent magnetization along $\langle 110\rangle$, which results from a strong uniaxial anisotropy at the stage of very thin Fe coverage. This direction changes to one of the $\langle 100\rangle$ directions with increasing film thickness. In both cases this is due to the thick Fe films beginning to show bulk bcc Fe features. These findings are consistent with the findings of Bland and co-workers. ${ }^{14}$

We now turn our attention to the valence structure of the Fe films. Figures 3(a) and 3(b) are the results for a $90 \AA \mathrm{Fe}$ film on a vicinal, Ga-rich substrate (sample B), and Figs. 3(c) and 3(d) are for an $80 \AA$ Fe film on a singular Ga-rich substrate (sample D). While the spin polarizations [Figs. 3(a) and 3(c)] of both samples exhibit a "dip" at around $1.5 \mathrm{eV}$ binding energy similar to that observed generally for $\mathrm{Fe}$ films, the variation in spin polarization shown by the $\mathrm{Fe}$ on the vicinal substrate (B) is more extreme than that exhibited by $\mathrm{Fe}$ on the singular substrate (D). In addition, both samples exhibit positive polarizations at $E_{f}$. Figures $3(\mathrm{~b})$ and $3(\mathrm{~d})$ give the spin-resolved valence photoemission spectra that display a clear difference between the majority spin and minority spin. Interestingly, the double-peak structure in the majority-spin channel, which arises due to splitting in the Fe cubic crystal field, ${ }^{15}$ is more pronounced for the Fe film on the vicinal substrate [Fig. 3(b)] than for Fe on the singular substrate [Fig. 3(d)]. A pronounced splitting was also found for an Fe thickness of only $50 \AA$ on a vicinal substrate. The enhancement may well be an indication of better crystallographic quality of Fe films grown on vicinal surfaces, consistent with our LEED observations.

To summarize, we report preliminary details of the magnetic and electronic structure characterization of Fe films grown on vicinal $\mathrm{GaAs}(001)$ substrates. As indicated by LEED, these films exhibit enhanced order compared with films grown on singular GaAs substrates. The films were studied by spin-resolved and spin-integrated photoelectron spectroscopy, which clearly indicated that the substrate steps influence the growth behavior and the resultant magnetic properties of the films. The first indications are that the vicinal film magnetic properties are much less sensitive than their singular analogues to the precise stoichiometry of the initial GaAs substrate surfaces, which is important for process control in a device fabrication environment. Pronounced peak splitting in the spin-resolved valence photoemission of vicinal $\mathrm{Fe}$ samples also suggests that the films are of high quality.

The authors gratefully acknowledge the financial support of the EPSRC for a research grant and for the scholarships awarded to two of the authors (T.Z. and M.S.) by the EPSRC, ORS, the University of Leeds, and the University of Salford. The authors also thank Dr. R. Grey and Dr. M. Hopkinson, the University of Sheffield, who provided them with the GaAs wafers used in this work.

${ }^{1}$ E. H. Rhoderick and R. H. Williams, Metal-Semiconductor Contacts (Clarendon, Oxford, U.K., 1988).

${ }^{2}$ S. Datta and B. Das, Appl. Phys. Lett. 56, 665 (1990).

${ }^{3}$ S. A. Chambers, F. Xu, H. W. Chen, I. M. Vitomirov, S. B. Anderson, and J. H. Weaver, Phys. Rev. B 34, 6605 (1986).

${ }^{4}$ A. Filipe and A. Schuhl, J. Appl. Phys. 81, 4359 (1997).

${ }^{5}$ M. Zölfl, M. Brockmann, M. Köhler, S. Kreuzer, T. Schweinböck, S. Miethaner, F. Bensch, and G. Bayreuther, J. Magn. Magn. Mater. 175, 16 (1997).

${ }^{6}$ M. Brockmann, M. Zölfl, S. Miethaner, and G. Bayreuther, J. Magn. Magn. Mater. 198-199, 384 (1999).

${ }^{7}$ T. Leeb, M. Brockmann, F. Bensch, S. Miethaner, and M. Bayeuther, J. Appl. Phys. 85, 4964 (1999).

${ }^{8}$ B. A. Joyce, J. H. Neave, J. Zhang, D. D. Vvedensky, S. Clarke, K. J. Hugill, T. Shitara, and A. K. Myers-Beaghton, Semicond. Sci. Technol. 5, 1147 (1990).

${ }^{9}$ T. Kawamura, J. Maruta, and A. Ishii, Jpn. J. Appl. Phys., Part 1 39, 4376 (2000).

${ }^{10}$ J. M. Gaines, P. M. Petroff, H. Kroemer, R. J. Simes, R. S. Geels, and J. H. English, J. Vac. Sci. Technol. B 6, 1373 (1998).

${ }^{11}$ M. Spangenberg, T. Zhang, N. Takahashi, T.-H. Shen, D. Greig, S. Cornelius, E. A. Seddon, and J. A. D. Matthew (unpublished).

${ }^{12}$ B. Sinkovic, E. Shekel, and S. L. Hulbert, Phys. Rev. B 52, R8696 (1995).

${ }^{13}$ Y. B. Xu, D. Greig, E. A. Seddon, and J. A. D. Matthew, Phys. Rev. B 55, 11442 (1997).

${ }^{14}$ M. Gester, C. Daboo, R. J. Hicken, S. J. Gray, A. Erole, and J. A. Bland, J. Appl. Phys. 80, 347 (1996).

${ }^{15}$ V. C. Moruzzi, J. F. Janak, and A. R. Williams, Calculated Electronic Properties of Metals (Pergamon, New York, 1978). 